\title{
CRESCIMENTO DOS FRUTOS DE LARANJEIRA 'SALUSTIANA' SITUADOS EM RAMOS ANELADOS COM DIVERSAS RELAÇÕES DE FOLHAS/FRUTOS'
}

\author{
DALMO LOPES DE SIQUEIRA² ${ }^{2}$ JOSÉ LUIZ GUARDIOLA ${ }^{3}$ EMANUEL FERNANDO MAIA DE SOUZA ${ }^{4}$
}

RESUMO- A relação entre a área foliar e o crescimento dos frutos é um tema que freqüentemente recebe a atenção dos pesquisadores, por influenciar diretamente na produtividade das plantas e na qualidade dos frutos. Neste trabalho, avaliou-se o efeito da área foliar sobre o crescimento dos frutos da laranjeira 'Salustiana'. Foram utilizados ramos com 12 meses de idade e com apenas um fruto terminal. Os ramos foram anelados visando a manter diversas relações de folhas/fruto. Avaliaram-se, semanalmente, o crescimento dos frutos e os teores de amido presentes nas folhas durante um período de 42 dias. O crescimento dos frutos, avaliado na "fase de crescimento II", dependeu da área foliar disponível por fruto, sendo que 30 folhas foram suficientes para garantir o seu crescimento. As reservas de amido nas folhas dependeram da área foliar disponível por fruto e reduziram à medida que os frutos apresentaram aumentos no diâmetro e nas massas fresca e seca.

Termos para indexação: relações fonte-dreno, Citrus sinensis (L.) Osbeck, amido

\section{GROWTH OF THE FRUITS OF 'SALUSTIANA' SWEET ORANGE LOCATED IN GIRDLED SHOOTS WITH SEVERAL LEAVES TO FRUIT RATIOS}

\begin{abstract}
The relationship between the foliar area and the fruit growth is an important theme because affects the tree productivity and fruits quality. In this work was evaluated the effect of the foliar area on the growth of the Salustiana's sweet orange fruits. Girdled shoots of 12 months were used with a single terminal fruit and several leaves-fruit ratios. It was evaluated, weekly, the fruit growth and the leaves starch contents during 42 days. The fruit growth, evaluated in the stage II, depended on the available leaf area per fruit, provided that 30 leaves were enough to guarantee its growth. The starch reserves in the leaves depended on the available leaf area per fruit and they reduced with the increase in the diameter and dry and fresh weight of the fruits.
\end{abstract}

Index terms: source-sink relationships, girdling, Citrus sinensis (L.) Osbeck, starch

\section{INTRODUÇÃO}

A laranjeira 'Salustiana', provavelmente, originou-se de uma mutação da laranjeira 'Comuna'. É uma árvore de tamanho médio com tendência a crescer verticalmente. Seus frutos são sem sementes, de tamanho médio a grande, e possuem casca ligeiramente rugosa (Agustí, 2000). No Brasil, essa variedade encontra-se em fase de avaliação (Radmann \& Oliveira, 2003).

As interações fisiológicas existentes entre os órgãos vegetais capazes de exportar carboidratos (fontes) e os órgãos que demandam estes compostos (drenos) são chamadas relações fonte-dreno (Kozlowski \& Pallardy, 1997). Estas relações, fontedreno, são importantes, pois influenciam na produção das plantas e no tamanho dos frutos (Minchin et al., 1997). Pesquisas realizadas com várias espécies frutíferas mostram que há uma relação inversa entre o número de frutos por planta e o seu tamanho, sendo que qualquer modificação no número de frutos na planta implica também alterações no tamanho (Yeshitela et al., 2004; Lakso et al., 2001; Guardiola \& García-Luis, 2000; Costa \& Vizzotto, 2000).

Em geral, acredita-se que o tamanho da área foliar fotossinteticamente ativa, disponível para cada fruto, é o principal fator responsável pelo seu tamanho, embora a eficiência fotossintética das folhas e a distribuição espacial dos frutos na planta também sejam importantes (Fishler et al., 1983). Entretanto, a atividade fotossintética das folhas não é constante, havendo um mecanismo de controle da oferta-demanda de carboidratos, que pode restringir a atividade fotossintética, na ausência de drenos (Fishler, 1983; Azcon-Bieto, 1983; Paul \& Foyer, 2001; Sawada et al., 2001).

Dados científicos sobre o efeito das relações fonte-dreno nas plantas frutíferas são úteis, pois permitem melhor conhecimento dos processos biológicos e fisiológicos que controlam a produção e fundamentam determinadas práticas culturais, como, por exemplo, a poda e o desbaste de frutos (Famiani et al., 2000).

A técnica da incisão anelar consiste no corte da casca do ramo ou tronco da planta em toda a sua circunferência. Esse tratamento, que não remove qualquer parte da casca, interrompe o transporte via floema, favorecendo o acúmulo de carboidratos nas folhas acima da região anelada, enquanto os frutos (drenos) os reduzem (Cohen, 1981; Iwahori et al., 1990; Sartori \& Ilha, 2005). Com base nessas informações, é possível controlar as relações fonte-dreno em uma planta, utilizando diferentes relações folhas/ frutos em ramos anelados.

$\mathrm{O}$ anelamento, por sua vez, é a retirada de um anel completo da casca (epiderme, capas subepidérmicas e floema) do tronco ou de ramos de árvores, impedindo o transporte da seiva do floema

(Trabalho 061-06). Recebido em: 10-05-2006. Aceito para publicação em: 14-03-2007.

${ }^{2}$ Prof.Dr. Departamento de Fitotecnia, Universidade Federal de Viçosa, 36570-000, Viçosa - MG. Email: siqueira@ufv.br

${ }^{3}$ Prof.Dr. Departamento de Biologia, Universidad Politécnica de Valencia - Valencia - Espanha. Email: jlguardiola@bvg.upv.es

${ }^{4}$ Mestrando em Fitotecnia, Departamento de Fitotecnia, Universidade Federal de Viçosa, 36570-000, Viçosa - MG. Email: emanuelfms@yahoo.com.br. 
para as demais partes da planta (Sartori \& Ilha, 2005). Desta forma, além do acúmulo de carboidratos, também há o acúmulo de fitorreguladores (auxinas, giberelinas, citocininas, ácido abscíssico) e de outros metabólitos (Cutting \& Line, 1993; Xianjun et al., 1999). Esse efeito pode dificultar a extrapolação dos resultados obtidos de ramos anelados para plantas em condições naturais, sem anelamento; entretanto, como o anelamento geralmente é realizado da mesma forma em todas as plantas, é válido comparar tratamentos com várias relações de folhas/frutos.

O objetivo do presente trabalho foi avaliar o efeito de diferentes relações de fonte-dreno sobre o crescimento de frutos e teores de carboidratos nas folhas de laranjeiras 'Salustiana' situadas em ramos anelados.

\section{MATERIAL E MÉTODOS}

O experimento foi conduzido durante o período de 15-062000 a 27-07-2000, em propriedade particular, na cidade de Valência - Espanha, em um pomar de laranjeiras 'Salustiana' e enxertadas sobre citrange 'Carrizo'. As plantas estavam com aproximadamente 30 anos de idade e foram irrigadas por gotejamento. Como o experimento foi conduzido em pomar comercial, a adubação foi controlada pelo produtor e realizada de acordo com análises foliares e de solo, por fertirrigação.

Foram utilizados cinco tratamentos: 1 - Controle (ramo com apenas um fruto terminal, sem anelamento); 2 - Presença de um único fruto no ramo, com anelamento realizado abaixo de 40 folhas; 3 - Presença de um único fruto no ramo, com anelamento realizado abaixo de 30 folhas; 4 - Presença de um único fruto no ramo, com anelamento realizado abaixo de 20 folhas; 5 - Presença de um fruto no ramo, com anelamento realizado abaixo de 10 folhas.

Foram selecionados cinqüenta frutos com diâmetro em torno de $30 \mathrm{~mm}$ ( $\pm 0,2 \mathrm{~mm})$, em nove plantas, situados em ramos contendo apenas um fruto terminal (inflorescência unifloral). Utilizaram-se 10 frutos por tratamento, considerando cada fruto como uma repetição, usando o delineamento inteiramente casualizado.

Semanalmente, foram avaliados o diâmetro dos frutos e os teores de amido nas folhas, usando uma folha por tratamento, colhidas imediatamente acima do local onde foi feito o anelamento. $\mathrm{O}$ amido foi determinado pelo método antrona-sulfúrico, de McCready et al, (1950).

A partir do dia 29-06-2000, foram coletados 25 frutos, em ramos situados em plantas fora do experimento, cujos diâmetros estavam compreendidos entre o menor e maior diâmetro avaliado nos frutos do experimento. Nestes frutos, foram avaliados o diâmetro ( $\mathrm{mm})$, a massa fresca e a massa seca após secagem em estufa de circulação forçada à temperatura de $70^{\circ} \mathrm{C}$. Essas informações foram usadas para estimar a massa seca dos frutos do experimento, usando equações de regressão lineares (Tabela 1).

Ao final do experimento (27-07-2000), os ramos foram coletados, sendo cortados no local onde foi realizado o anelamento. Nesses ramos, foram avaliados o número de folhas, área foliar, massa seca e fresca e teores de carboidratos das folhas. Nos frutos, avaliaram-se o diâmetro e a massa fresca e seca.
Os dados das características avaliadas foram submetidos à análise de variância, seguida do ajuste de regressão linear, utilizando o software livre R (R DEVELOPMENT CORE TEAM, 2004), fundamentando a escolha do modelo na dispersão dos resíduos, nos testes de significância dos parâmetros e do coeficiente de regressão.

\section{RESULTADOS E DISCUSSÃO}

A área foliar e o diâmetro do pedúnculo dos frutos, avaliados nos ramos que receberam os diferentes tratamentos, encontram-se na Tabela 2. Para o controle, não foi avaliada a área foliar em virtude de não haver um ponto de referência (anelamento) a partir do qual as folhas seriam coletadas.

Verifica-se que, para o tratamento "40 folhas/fruto", a área foliar foi $608,6 \%$ superior à área do tratamento " 10 folhas/fruto" (443,65 cm² e 62,61 $\mathrm{cm}^{2}$, respectivamente). O diâmetro do pedúnculo dos frutos diminuiu à medida que houve redução na área foliar $(\mathrm{r}=$ 0,99). García-Luís et al., 2002, observaram que a área transversal do xilema no pedúnculo dos frutos cítricos está diretamente correlacionada com o diâmetro dos frutos. Essa correlação devese, provavelmente, ao fato de que os pedúnculos com maior diâmetro também apresentaram maior área do xilema, que foi avaliada em corte transversal do pedúnculo (Tabela 2).

A evolução do diâmetro e das massas fresca e seca dos frutos ao longo do tempo foi representada por equações de regressão quadrática (Figuras 1; 2 e 3). Essas características aumentaram ao longo do tempo; entretanto, a magnitude do aumento dependeu do número de folhas presentes no ramo. Essa forte relação entre o crescimento dos frutos e a área foliar demonstra que o crescimento dos frutos depende da taxa fotossintética, que é controlada pela demanda dos frutos (GarcíaLuís et al., 2002).

Como o experimento foi iniciado quando os frutos estavam com diâmetro médio de $30 \mathrm{~mm}$ e conduzido por um período de 42 dias, a avaliação do seu desenvolvimento ocorreu quando estes se encontravam na fase II do crescimento. O crescimento dos frutos cítricos é do tipo sigmoidal simples, dividido em três fases (Bain, 1958). Na fase I, são formadas praticamente todas as células dos frutos (divisão celular) e tem duração de 1,0 a 1,5 mês, dependendo da cultivar, do clima e dos tratos culturais. A fase II é caracterizada por um rápido crescimento do fruto, devido ao aumento do volume celular, havendo rápidas mudanças morfológicas e fisiológicas, e ausência de divisão celular. A fase III é marcada pela maturação dos frutos, na qual praticamente não há crescimento do endocarpo, todavia há aumento do conteúdo de sólidos solúveis totais, redução da acidez e possível alteração da pigmentação do flavedo. $\mathrm{O}$ crescimento rápido do fruto foi observado neste trabalho, sendo constatados aumentos de até $52,3 \%$ no diâmetro, durante o período de avaliação (tratamento 40 folhas/fruto).

O diâmetro dos frutos e massas seca e fresca dos frutos, no último dia do experimento, para os tratamentos-controle, 40 folhas/fruto e 30 folhas/fruto, não diferiram estatisticamente entre si. Para os tratamentos 10 folhas/fruto e 20 folhas/fruto, os diâmetros foram estatisticamente iguais e inferiores $(p<0,05)$ aos 
dos demais tratamentos. As massas fresca e seca dos frutos foram menores para o tratamento 10 frutos/folha, que diferiu de todos os demais (Tabela 3).

Esses resultados indicam que 30 folhas (área de 290,21 $\mathrm{cm}^{2}$ ) foram suficientes para suportar o crescimento dos frutos, sem qualquer diferença estatística quando comparada com a presença de maior número de folhas/fruto.

Como as avaliações do crescimento dos frutos ocorreram durante um curto período, tornou-se difícil comparar este resultado com o de outros autores, pois não foi possível inferir se o número mínimo de 30 folhas/fruto seria suficiente para sustentar o crescimento dos frutos até a maturação.

Siqueira et al. (2000), observaram crescimento linear no diâmetro de laranjas 'Hamlin', avaliado a partir de $33 \mathrm{~mm}$ até a colheita dos frutos, em função do número de folhas presentes, que variou de 50 a 200 folhas/fruto. Esse resultado demonstrou que os frutos teriam potencial para alcançar maiores diâmetros, caso houvesse disponibilidade de mais folhas/fruto.

A relação folhas/fruto ideal para o máximo crescimento dos frutos é variável, pois depende da espécie, do local de cultivo e da atividade fotossintética das folhas. Para o pomeleiro (Citrus paradisi Macfad), em plantas adultas, foi encontrada uma relação em torno de $0,3 \mathrm{~m}^{2}$ de folhas por fruto (Fishler et al., 1983). No entanto, para a castanheira portuguesa (Castanea sativa Mill.), são necessárias aproximadamente cinco folhas/fruto para assegurar um desenvolvimento normal do fruto (Famiani et al., 2000) e para a noz macadâmia (Macadamia integrifolia Maiden \& Betche), aproximadamente 50 folhas são necessárias (Trueman \& Turnbull, 1994).

Os teores de amido (Figura 4), em geral, reduziram-se com o tempo para todos os tratamentos, entretanto maiores reduções foram observadas no tratamento com menor relação folha/fruto (10 folhas/fruto), cujo teor diminuiu de $7,32 \pm 0,8 \%$ para $3,40 \pm$ $0,14 \%$. Com exceção desse tratamento, todos os demais apresentaram concentrações semelhantes de amido ao final do experimento, indicando que a taxa fotossintética e o subseqüente acúmulo de amido são controlados pela força de dreno exercida pelos frutos (Paul \& Foyer, 2001).

TABELA 1 - Equações de regressão linear utilizadas para calcular as massas fresca e seca dos frutos do experimento, a partir dos diâmetros medidos semanalmente.

\begin{tabular}{|c|c|c|c|c|}
\hline Data & Massa Fresca & $R^{2}$ & Massa Seca & $R^{2}$ \\
\hline $29-06-00$ & $\begin{array}{l}\hat{Y}=21,5076+ \\
0,628603 * * X\end{array}$ & 0,93 & $\begin{array}{l}\hat{Y}=20,8164+ \\
2,8818^{* *} \mathrm{X}\end{array}$ & 0,88 \\
\hline 06-07-00 & $\begin{array}{c}\hat{\mathrm{Y}}=24,1167+ \\
0,508411^{* *} \mathrm{X}\end{array}$ & 0,97 & $\begin{array}{l}\hat{Y}=23,0948+ \\
2,40952 * * X\end{array}$ & 0,95 \\
\hline $13-07-00$ & $\begin{array}{l}\hat{Y}=25,1054+ \\
0,477567 * * X\end{array}$ & 0,96 & $\begin{array}{l}\hat{Y}=23,2438+ \\
2,39578^{* *} \mathrm{X}\end{array}$ & 0,87 \\
\hline $20-07-00$ & $\begin{array}{l}\hat{Y}=27,4469+ \\
0,400994 * * X\end{array}$ & 0,97 & $\begin{array}{l}\hat{Y}=25,2516+ \\
2,07268 * * X\end{array}$ & 0,93 \\
\hline
\end{tabular}

TABELA 2 - Valores médios da área foliar e diâmetro do pedúnculo dos frutos, com seus respectivos desvios-padrão, determinados no final do experimento (27-07-2000).

\begin{tabular}{ccc}
\hline Tratamentos & $\begin{array}{c}\text { Área foliar } \\
\mathrm{cm}^{2}\end{array}$ & $\begin{array}{c}\text { Diâmetro do } \\
\text { pedúnculo dos frutos } \\
(\mathrm{mm})\end{array}$ \\
\hline Controle & - & $3,32 \pm 0,33$ \\
40 Folhas/fruto & $443,65 \pm 42,02$ & $3,47 \pm 0,04$ \\
30 Folhas/fruto & $290,21 \pm 57,35$ & $3,27 \pm 0,01$ \\
20 Folhas/fruto & $179,41 \pm 34,10$ & $2,98 \pm 0,00$ \\
10 Folhas/fruto & $62,61 \pm 1,80$ & $2,81 \pm 0,06$ \\
\hline
\end{tabular}

TABELA 3 - Características do crescimento de laranjas 'Salustiana', avaliadas no final do experimento (27-07-2006).

\begin{tabular}{cccc}
\hline \multirow{2}{*}{ Tratamentos } & \multicolumn{3}{c}{ Características } \\
\cline { 2 - 4 } & $\begin{array}{c}\text { Diâmetro } \\
\text { dos frutos }(\mathrm{mm})\end{array}$ & $\begin{array}{c}\text { Massa fresca } \\
\text { dos frutos }(\mathrm{g})\end{array}$ & $\begin{array}{c}\text { Massa seca } \\
\text { dos frutos }(\mathrm{g})\end{array}$ \\
\hline Controle & $45,5 \mathrm{a}$ & $45,6 \mathrm{a}$ & $9,9 \mathrm{a}$ \\
40 folhas & $45,7 \mathrm{a}$ & $46,4 \mathrm{a}$ & $9,6 \mathrm{a}$ \\
30 folhas & $44,3 \mathrm{a}$ & $41,8 \mathrm{a}$ & $8,9 \mathrm{a}$ \\
20 folhas & $41,5 \mathrm{~b}$ & $33,3 \mathrm{~b}$ & $6,9 \mathrm{~b}$ \\
10 folhas & $37,6 \mathrm{~b}$ & $25,5 \mathrm{c}$ & $5,1 \mathrm{c}$ \\
\hline
\end{tabular}

Médias seguidas de mesmas letras nas colunas não diferem entre si, pelo teste de Tukey $(\mathrm{p}<0,05)$

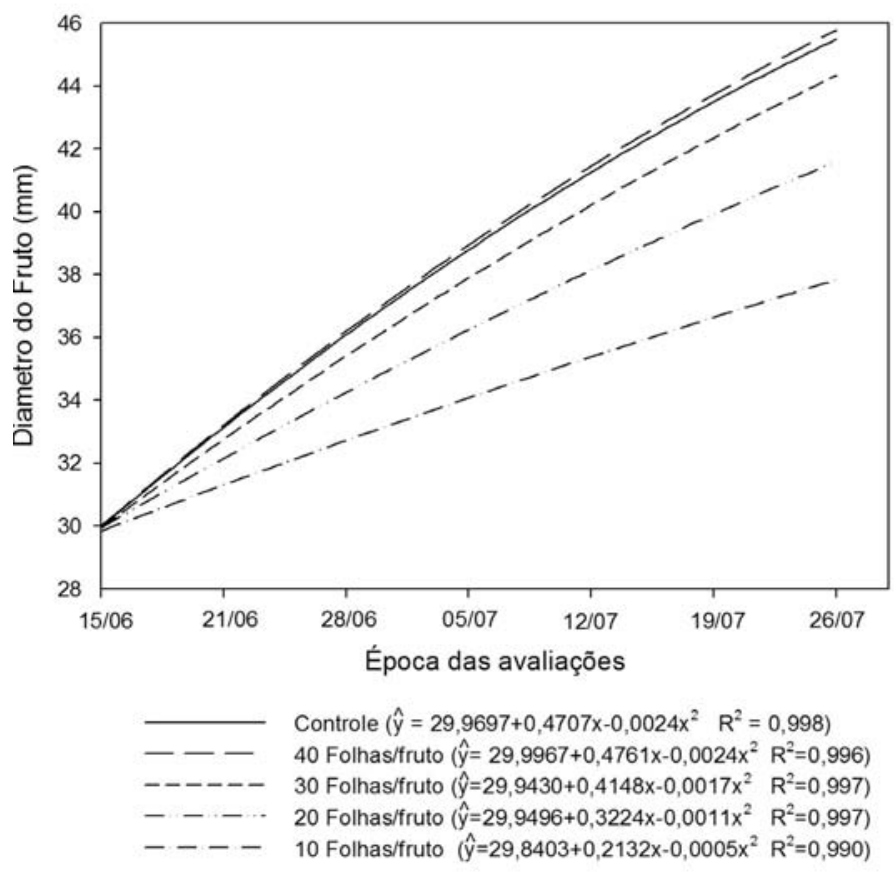

FIGURA 1 - Efeito da relação folhas/frutos sobre o diâmetro dos frutos $(\mathrm{mm})$, situados em ramos anelados de laranjeira 'Salustiana' (29-06-2000 a 27-072000). 


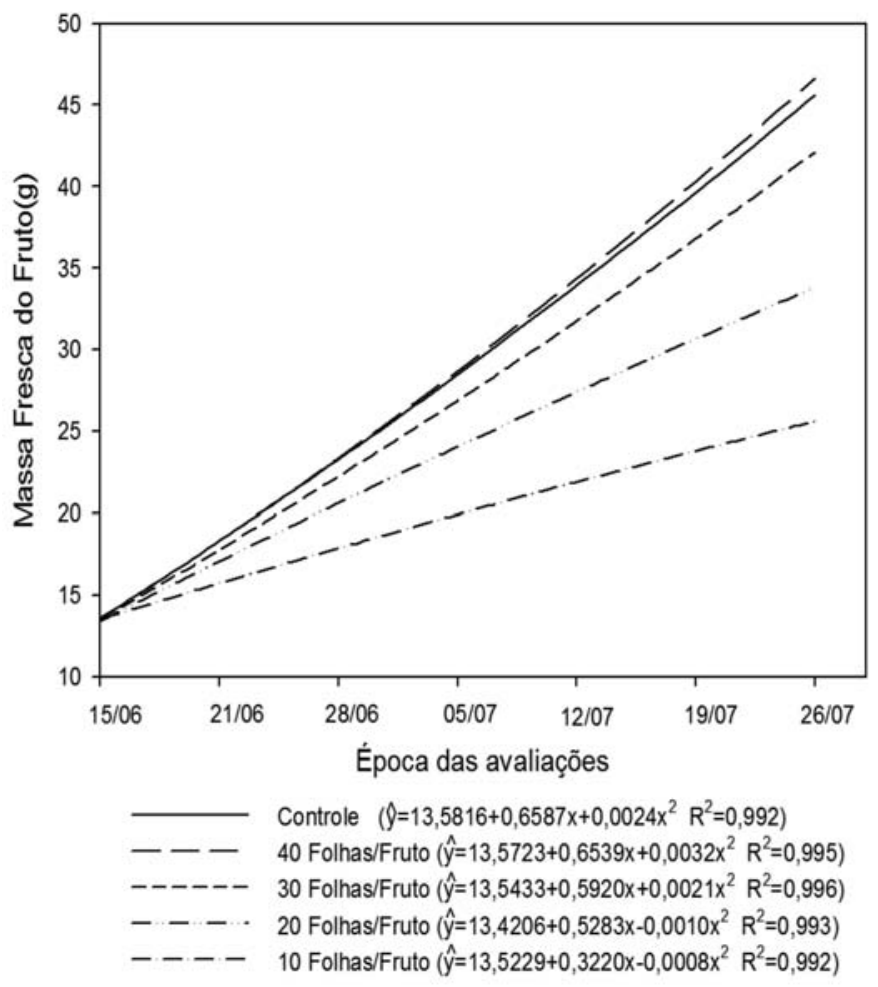

FIGURA 2 - Massa fresca dos frutos de laranjeiras 'Salustina', situados em ramos anelados, com diferentes relações folha/fruto (29-06-2000 a 27-07-2000).

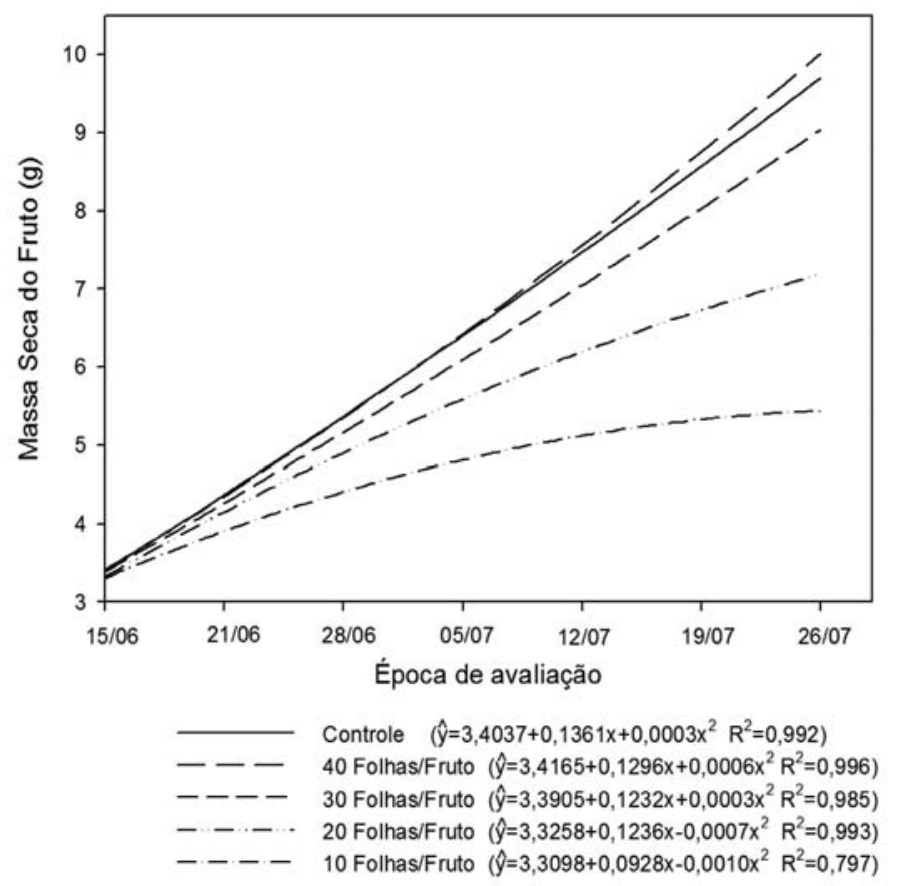

FIGURA 3 - Massa seca dos frutos de laranjeiras 'Salustina', situados em ramos anelados, com diferentes relações folha/fruto (29-06-2000 a 27-07-2000).

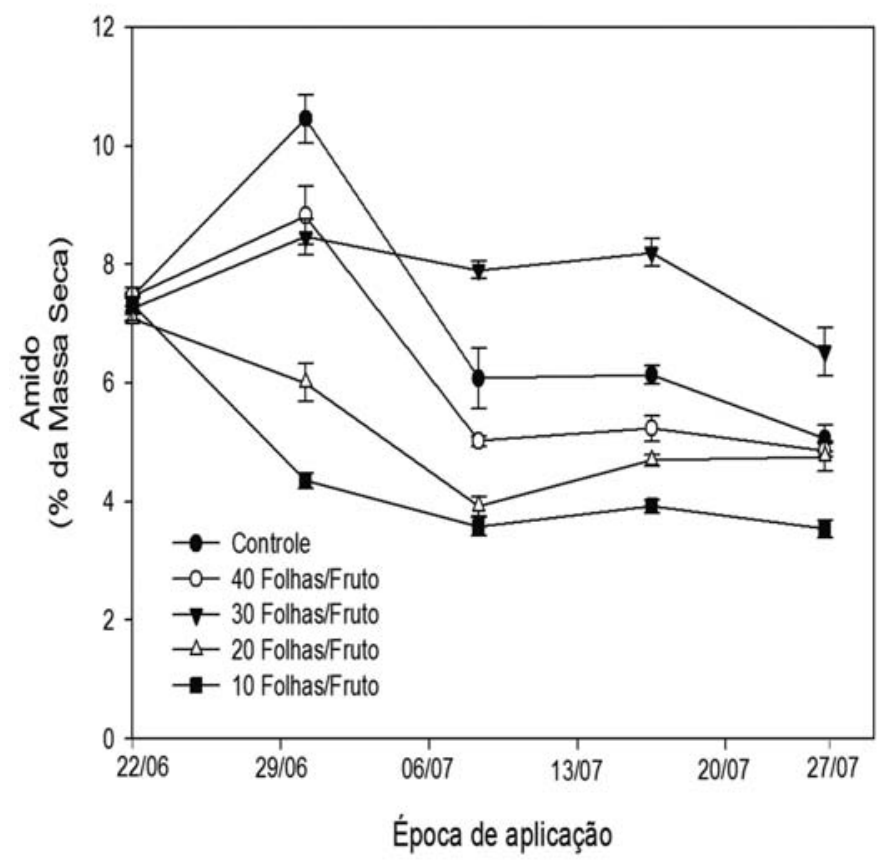

FIGURA 4 - Variação nos teores de amido em folhas de laranjeiras 'Salustiana', situados em ramos anelados, com diferentes relações folha/fruto (29-06-2000 a 27-07-2000).

\section{CONCLUSÕES}

1- O crescimento de laranjas 'Salustiana', avaliado durante 42 dias na "fase de crescimento II", dependeu da área foliar disponível por fruto. Nessa etapa, 30 folhas foram suficientes para garantir o crescimento dos frutos.

2- As reservas de amido nas folhas dependeram da área foliar disponível/fruto e reduziram à medida que os frutos apresentaram aumentos no diâmetro e nas massas fresca e seca.

\section{REFERENCIAS}

AGUSTÍ, M. Citricultura. Madrid: Mundi-Prensa, 2000. 416p.

AZCON-BIETO, J. Inhibition of photosynthesis by carbohydrates in wheat leaves. Plant Physiology, Rockville, v.73, n.3,p. 681-686, 1983.

BAIN, J. M. Morphological, anatomical, and physiological changes in the developing fruit of the Valencia orange, Citrus sinensis (L) Osbeck. Australian Journal of Botany, Collingwood, v.6, n.1, p.1-23, 1958.

COHEN, A. Recent developments in girdling of citrus trees. Proceedings of the International Society of Citriculture, Washington, v.1, p.196-199, 1981.

COSTA, G.; VIZZOTTO, G. Fruit thinning of peach trees. Plant Growth Regulation, Dordrecht, v.31, n.1-2, p. 113-119, 2000.

CUTTING, J. G. M.; LINE, M. C. Girdling and the reduction in shoot xylem sap concentration of cytokinins and gibberellins in peach. Journal of Horticultural Science, Ashford, v.68, n., p.619-626, 1993. 
FAMIANI, F.; PROIETTI, P.; PALLIOTTI, A.; FERRANTI, F. ANTOGNOZZI, F. Effects of leaf to fruit ratios on fruit growth in chestnut. Scientia Horticulturae, Amsterdam, v.85, 1-3 p.145-152, 2000.

FISHLER, M.; GOLSCHMIDT, E.; MONSELISE, S. P. Leaf area and fruit size on girdled grapefruit. Journal of the American Society for Horticultural Science, Alexandria, v.108, n.102, p.218-221, 1983.

GARCÍA-LUIS, A.; OLIVEIRA, M.E.M.; BORDÓN, Y.; SIQUEIRA, D.L.; TOMINAGA, S.; GUARDIOLA, J. L. Dry Matter Accumulation in citrus fruit is not limited by transport capacity of the pedicel. Annals of Botany, London, v.90, n. 6, p. 755-764, 2002.

GUARDIOLA, J.L.; GARCÍA-LUIS, A. Increasing fruit size in Citrus. Thinning and stimulation of fruit growth. Plant Growth Regulation, Dordrecht, v.31, p.121-132, 2000.

IWAHORI, S.; GARCÍA-LUIS, A.; SANTAMARINA, P.; MONERI, C.; GUARDIOLA, J. L. The influence of ringing on bud development and flowering in Satsuma mandarin. Journal of Experimental Botany, London, v.41, n.231, p.1.3411.346, 1990.

KOZLOWSKI, T. T.; PALLARDY, G. S. Physiology of woody plants. $2^{\text {nd }} e d$. San Diego: Academic Press, 1997. 411p.

LAKSO, A. N.; ROBINSON, T. L.; GOFFINET, M. C.; WHITE, M. D. Apple fruit growth responses to varying thinning methods and timing. Acta Horticulturae, Wageningen, v.557, p. 407412, 2001.

McCREADY, R. M.; GUGGOLZ, J.; SILVEIRA, V.; OWENS, H. S. Determination of starch an amylase in vegetables. Application to peas. Analytical Chemistry, Washington, v.22, n.9, p.1.156-1.158, 1950.

MINCHIN, P. E. H.; THORPE, M. R.; WUNSCHE, J. N.; PALMER; J. W.; PICTON R. F. Carbon partitioning between apple fruits: short- and long-term response to availability of photosynthate Journal of Experimental Botany, London, v.48, n.7, p.1.401-1.406, 1997.

PAUL, M. J.; FOYER, C. Sink regulation of photosynthesis. Journal Experimental of Botany, London, v.52, n. 360, p.1.383-1.400, 2001.

R DEVELOPMENT CORE TEAM. R: a language and environment for statistical computing. Vienna, Áustria: Foundation for Statistical Computing, 2004. Disponível em: <http://www.rproject.org >. Acesso em: set. 2004.

RADMANN, E. B.; OLIVEIRA, R. P. de. Caracterização de cultivares apirênicas de citros de mesa por meio de descritores morfológicos. Pesquisa Agropecuária Brasileira, Brasília, v.38, n.9, p.1.123-1.129, 2003.

SARTORI, I. A.; ILHA, L. L. H. Anelamento e incisão anelar em fruteiras de caroço. Ciência Rural, Santa Maria, v.35, n.3, p.724-729, 2005.

SAWADA, S.; KUNINAKA, M.; WATANABE, K.; SATO, A.; KAWAMURA, H.; KOMINE, K.; SAKAMOTO, T.; KASAI, M. The mechanism to suppress photosynthesis through end-product inhibition in single rooted soybean leaves during acclimation to $\mathrm{CO}_{2}$ enrichment. Plant Cell Physiology,
Tokyo, v.42, n.10, 1.093-1.102, 2001.

SIQUEIRA, D. L. de; PEREIRA, W. E.; SALOMÃO, L. C. C.; BENEDITO, V.A. Características físicas e químicas do fruto da laranjeira 'Hamlin' em função do número de folhas e do anelamento. Revista Brasileira de Fruticultura, Jaboticabal, v.22, n., p.106-110, 2000.

TRUEMAN, S. J.; TURNBULL, C. G. N. Fruit set, abscission and dry matter accumulation on girdled branches of macadamia. Annals of Botany, London, v.74, n.6, p. 667-674, 1994.

XIANJUN, Z.; DEYAN, H.; HUIBAI, H.; DINGYAO, W. Carbohydrate and endohormone status in relation to fruit set as influenced by trunk spiral girdling of young litchi trees. Acta Horticulturae Sinica, Beijing, v.26, n. 2_p.77-80, 1999.

YESHITELA T.; ROBBERTSE, P.J.; FIVAS, J. Effects of fruit thinning on 'Sensation' mango (Mangifera indica) trees with respect to fruit quantity, quality and tree phenology. Experimental Agriculture,_Cambridge,_v.40,_n.4, p.433-444, 2004. 